\title{
串级萃取理论和稀土分离技术的发展趋势及相关 进展
}

廖春生 ${ }^{1,2 *}$, 程福祥 ${ }^{1,2}$, 吴声 ${ }^{1,2}$, 严纯华 ${ }^{2}$

1. 五矿(北京)稀土研究院有限公司, 北京 100085

2. 北京大学化学与分子工程学院, 稀土材料化学及应用国家重点实验室, 北京分子科学中心, 北京 100871

*通讯作者, E-mail: liao@cre-ol.com

收稿日期：2020-07-14; 接受日期：2020-08-31; 网络版发表日期：2020-10-22

摘要稀土被誉为“新材料宝库”. 我国是稀土资源大国, 稀土萃取分离理论和技术也处世界领先水平. 近 20 年 来, 为适应环境友好和智能生产要求, 我国的稀土萃取分离技术呈现了连续色层化、超高纯化、控制自动化和联 动一体化等发展趋势和特点. 联动萃取工艺的引入使萃取分离流程达到了连续离子交换色层法效果. 萃取过程中 交叉污染成因分析和控制技术的发展拓展了萃取分离提纯极限。基于全流程仿真优化设计专家系统的稀土分离 自动控制可有力保障分离产品的质量稳定. 萃取分离与相关工序的联动一体化是进一步提升稀土分离绿色化的 新思路. 本文结合作者多年来的研究工作, 从分离流程的连续色层化、分离产品的超高纯化、分离过程控制的自 动化和分离工序的联动一体化几个方面介绍了串级萃取理论和稀土分离技术的发展趋势及相关进展.

关键词串级萃取理论, 稀土分离, 超高纯稀土, 自动控制, 联动一体化

\section{1 引言}

稀土是国内外科学家尤其是材料专家最为关注的 一组元素，其工业发展水平直接影响着航空航天、核 能、电子、冶金、化工等诸多高新技术领域．我国不 仅稀土资源储量高居世界首位，稀土的分离提纯理论 与技术也领先世界.

国外稀土工业化分离提纯相关研究开发始于 20 世 纪初，早期采用烧碱分解、沉淀结晶分离工艺从独居 石中回收稀土. 1950年前，德国稀土产量占世界总产 量的 $95 \%$ ，之后美国开发出离子交换法分离稀土技术， 使稀土的纯度大幅提升 ${ }^{[1]}$. 我国稀土科技工作者从上
世纪50年代开始对溶剂萃取法分离稀土元素进行了大 量研究开发, 取得了许多科研成果, 如采用N263萃取 分离出纯度为 $99.99 \%$ 的氧化钎, 取代了高成本的离子 交换法分离氧化钇工艺，以及将氨化P507萃取分离稀 土和环烷酸萃取钎的工艺用于我国的稀土湿法冶金工 业等 ${ }^{[2]}$.

上世纪70年代，北京大学徐光宪院士等提出的串 级萃取理论 ${ }^{[3 \sim 8]}$ 及其工业实践引导了稀土分离科技和 产业的全面革新，我国的稀土萃取分离技术也由此进 入了快速发展期，并对国际稀土界形成了“中国冲击”. 串级萃取理论建立了稀土溶剂萃取分离工艺优化设计 计算方法, 并通过结合计算机技术, 逐步实现了理论设

引用格式: Liao CS, Cheng FX, Wu S, Yan CH. Trends and progress of the theory of countercurrent extraction and rare earth separation technology. Sci Sin Chim, 2020, 50: 1730-1736, doi: 10.1360/SSC-2020-0134 
计到实际工业生产应用的“一步放大”, ${ }^{[9,10]}$. 在该理论指 导下，稀土分离生产工艺从两出口逐步拓展到三出口 和多出口，适用范围也从恒定混合萃取比体系发展到 非恒定混合萃取比体系，基本实现了模拟计算进行稀 土串级萃取全分离工艺的参数设计和流程优化 ${ }^{[11 ~ 13]}$.

进入21世纪，工业制造业向环境友好和智能生产 转型升级的大背景对稀土萃取分离技术提出了更为高 端的发展要求. 在过去的 20 年间, 我国稀土分离技术已 呈现了流程连续色层化、产品超高纯化、过程控制自 动化和工序联动一体化等发展趋势和特点.

本文结合作者多年来的研究工作，从分离流程的 连续色层化、分离产品的超高纯化、分离过程控制的 自动化和分离工序的联动一体化几个方面介绍了串级 萃取理论和稀土分离技术的发展趋势及相关进展.

\section{2 分离流程的连续色层化}

1947年11月美国宣布用离子交换法分离稀土元素 获得成功 ${ }^{[1,14]}$, 自此离子交换色层技术开始用于单一 稀土元素的分离, 该法也成为当时能将所有稀土元素 制成高纯单一稀土化合物的唯一手段. 离子交换色层 法将交换剂、络合剂和淋洗剂联合使用, 可达到稀土 分离提纯的目的. 但该法只能间歇操作，且具有生产 周期长、产率低、投资大、成本高、单位单元处理量 小等缺点, 因而在稀土分离工业生产中逐渐被溶剂萃 取法所取代. 稀土分离是一个复杂的多组分分离过程, 但在离子交换色层法中，仅通过一个分离过程即可得 到所有组分的纯产品, 如图1所示; 但对于采用溶剂萃 取法的稀土全分离流程, 为得到各组分的纯产品, 常需 构建数个乃至数十个分离单元, 操作繁杂.

结合离子交换色层和溶剂萃取分离技术的优势, 实现稀土溶剂萃取分离流程的连续色层化、简化稀土 分离流程工艺操作和优化设计是近年稀土分离技术发 展的趋势之一. 2000 年以后, 基于串级萃取理论的计算 机仿真技术取得了重大突破. 通过建立一种新的串级 萃取仿真模型，发展了多组分多出口分馏萃取静态设 计和动态仿真技术 ${ }^{[15 \sim 17]}$, 结合可显著提升计算速度的 增强牛顿法编写的计算软件成为了稀土萃取分离新工 艺优化设计的有力手段, 也正是由此发展的联动萃取 分离工艺促进了稀土溶剂萃取分离流程的连续色 层化.

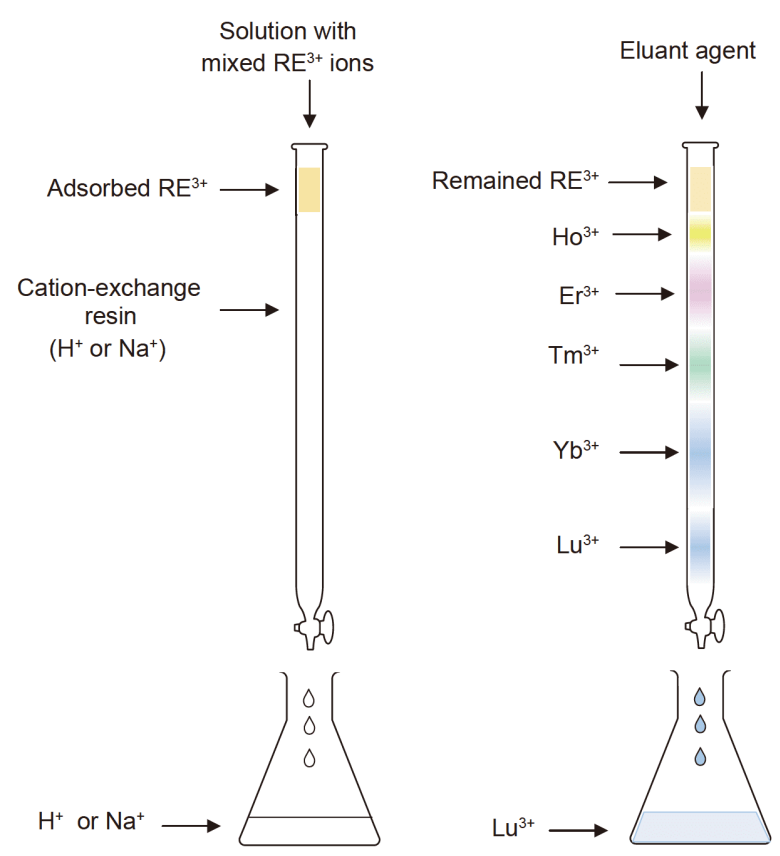

图 1 离子交换色层法分离稀土离子 (网络版彩图)

Figure 1 Separation of rare earth ions by ion exchange chromatography (color online).

联动萃取分离技术通过多组分稀土萃取分离流程 中相关分离单元间的横向和纵向衔接, 彼此提供萃取 有机相或洗涤液, 不仅可显著降低流程整体所需皇化 用碱和洗涤用酸消耗 ${ }^{[18-20]}$, 同时也简化了操作，相当 于将所有分离单元合并为一个单元的操作, 即类似于 离子交换色层法, 整体分离流程仅需一个料液输入 口、一个萃取有机相输入口和一个洗涤液输入口即可 由设置的各出口同时产出所有组分的纯产品, 且可实 现离子交换色层法不能实现的连续操作, 更适于工业 化规模生产. 以一个 $\mathrm{La} / \mathrm{Ce} / \mathrm{Pr} / \mathrm{Nd}$ 四组分稀土全分离为 例, 其联动萃取流程框架如图2所示, 其中含 $(\mathrm{LaCePr}) /$ $(\mathrm{CePrNd}) 、(\mathrm{LaCe}) /(\mathrm{CePr}) 、(\mathrm{CePr}) /(\mathrm{PrNd}) 、 \mathrm{La} / \mathrm{Ce} 、$ $\mathrm{Ce} / \mathrm{Pr} 、 \mathrm{Pr} / \mathrm{Nd}$ 六个分离单元. 图2中, 通过将六个分离 单元相互联动衔接, 只需在分离单元 $(\mathrm{LaCePr}) /$ $(\mathrm{CePrNd}) 、 \mathrm{La} / \mathrm{Ce}$ 和 $\mathrm{Pr} / \mathrm{Nd}$ 分别输入待分离的混合稀土 料液 $f$ 、萃取有机相 $S$ 和洗涤液 $W$, 即可由各纯产品出 口分别获得La、Ce、Pr和Nd的单一稀土溶液.

对于如图2所示的联动萃取分离流程, 目前可有两 种方法进行萃取有机相流量、洗涤液流量以及各分离 单元间联动衔接相关工艺参数的优化设计计算. 方法 一采用动态模拟仿真计算软件进行, 通过尝试不同参 


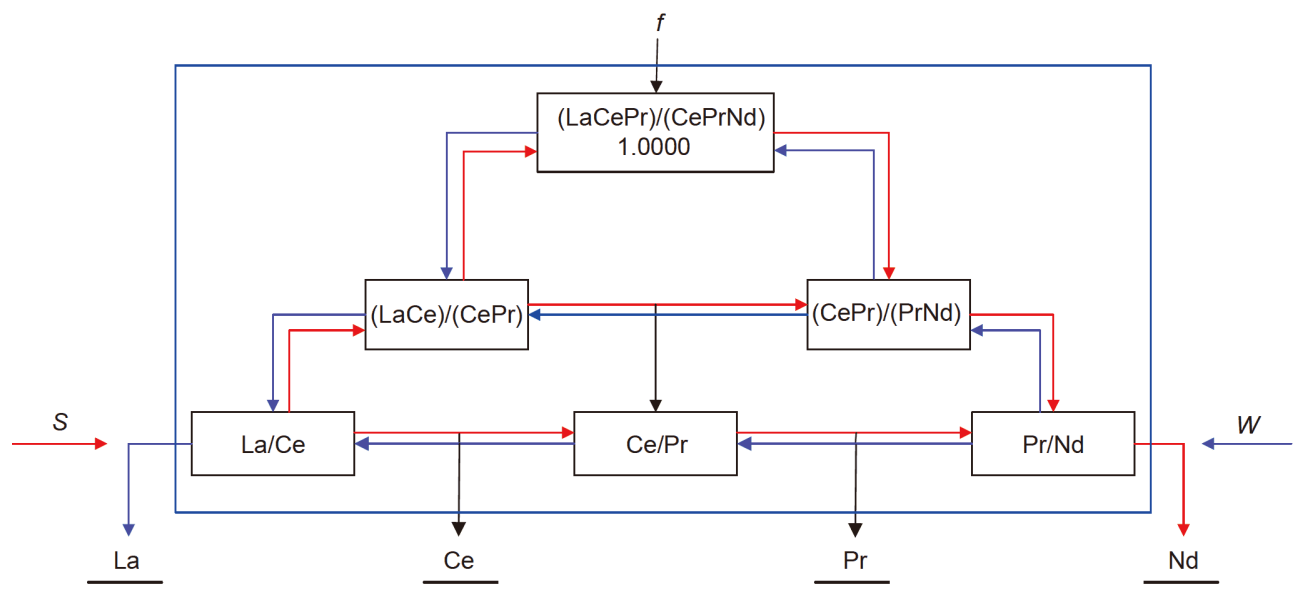

图 $2 \mathrm{La} / \mathrm{Ce} / \mathrm{Pr} / \mathrm{Nd}$ 联动萃取分离流程框架图 (红色、蓝色、黑色箭头分别代表有机相流、水相流、待确定) (网络版彩图) Figure 2 Flowsheet framework of $\mathrm{La} / \mathrm{Ce} / \mathrm{Pr} / \mathrm{Nd}$ hyperlink extraction separation (red, blue and black arrows represent organic, aqueous and dependent flows, respectively) (color online).

数组合计算所得产品的纯度. 但该法需从大量符合产 品纯度设计要求的计算结果中择优确定工艺参数, 工 作量大，且无法判定所选结果是否已最优化. 方法二 为通过近年发展的联动萃取分离工艺设计理论计算最 优化工艺参数 ${ }^{[21 ~ 26]}$. 该法可通过系列理论公式，直接 计算得到各种类型分离单元所需的理论最小萃取量 $S_{\min }$ 、最小洗涤量 $W_{\min }$ 以及分离单元间横向和纵向联 动衔接时的最优化流量, 最终计算得到流程整体所需 的 $S_{\min }$ 和 $W_{\text {min }}{ }^{[25]}$.

上述方法二设计的工艺流程为理论极限情况，实 际工业生产需要考虑不同产品结构、存槽投资等因 素, 可将两种方法结合使用, 方法一以方法二框定的边 界条件为基础进行优化设计. 图2流程中后端纯产品分 离层级依次进行 $\mathrm{La} / \mathrm{Ce} 、 \mathrm{Ce} / \mathrm{Pr}$ 和 $\mathrm{Pr} / \mathrm{Nd}$ 分离，槽体中各 组分随级数变化的分布曲线类似于离子交换色层技术 中的淋洗曲线, 如图3所示.

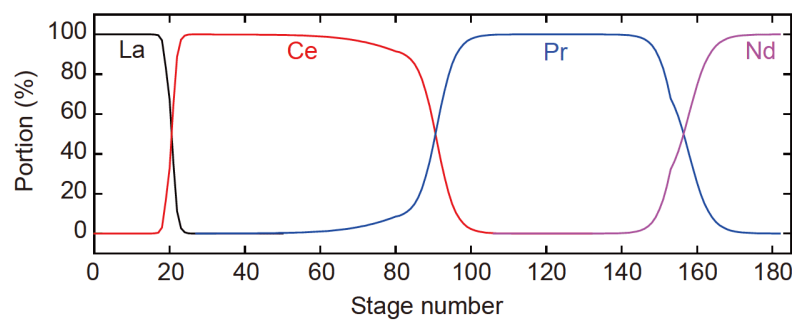

图 3 方法一设计流程纯产品分离层级槽体中各组分分布 图 (网络版彩图)

Figure 3 Component portion in the product cascade of the flow-sheet designed by Method I (color online).

\section{3 分离产品的超高纯化}

在稀土分离工业中，溶剂萃取方法一般可生产得 到纯度 $99 \%$ 至 $99.99 \%$ 的产品，一些位于萃取序列端头 的元素也可得到 $99.999 \%$ 以上产品，如P507体系中的 镧和镥、氧化还原体系中的铕和环烷酸体系中的钎. 而更高纯度的稀土产品一般需采用离子交换等方法 制备.

工业实践发现，溶剂萃取法制备更高稀土纯度产 品不仅需要解决相邻杂质元素的分离问题，还需解决 萃取序列端头杂质元素含量的控制问题. 相邻杂质元 素与目标超高纯产品的分离系数一般较小, 如铕/钝及 钇/铒等的分离系数都仅为 1.5 左右. 不过, 根据串级萃 取理论，通过对相关级数及处理量等工艺参数进行优 化, 即可得到满足相关产品高纯化要求的工艺设置.

萃取序列端头稀土杂质包括易萃组份杂质与难萃 组份杂质. 对于P507萃取体系, 易萃组份杂质主要是在 原料中广泛存在的镱、镥等难反萃重稀土杂质; 难萃 组份杂质一般为在萃取剂稀土㿝过程中易形成少量可 扰乱萃取顺序的微乳结构的镧等轻稀土杂质. 针对含 酸和多金属离子体系发展的非恒定混合萃取比体系串 级萃取理论 ${ }^{[27]}$ 可用于反萃取过程的优化设计，并计算 得到满足端头易萃组分杂质(如镥、镱)达到纯度要求 的反萃取工艺参数. 另外, 针对有机相中的微乳结构稳 定性进行了研究，开发出了稳定稀土㿝控制方法和破 乳技术. 
目前，应用上述相邻元素及萃取序列端头稀土杂 质控制技术开发出的超高纯稀土萃取分离工艺主要通 过两种形式实现. 一种是以特定目标稀土元素的富集 物为原料, 采用联动萃取技术, 从中间出口得到 99.999\%级以上的超高纯稀土产品. 以此方式已得到 99.999\% 99.9999\%级的Ce、 Tb、 Dy、 $\mathrm{Er} 、 \mathrm{Tm} 、 \mathrm{Yb}$ 等产品. 原则上全部稀土元素均可采用此工艺形式得 到超高纯稀土产品. 另一种工艺形式是以全组分混合 稀土料液为原料，在全分离流程中实现特定元素的超 高纯化生产. 目前已按此种工艺形式完成了 99.999 99.9999\%级 $\mathrm{Eu}$ 和 Gd的工业生产实践.

\section{4 分离过程控制的自动化}

稀土萃取分离工艺过程主要是通过控制有机相、 料液、洗涤液等物料的流量完成的. 随着相关领域的 技术进步，近年来稀土萃取分离过程的工业自动化水 平也在不断提高. 目前, 国内较为先进的稀土萃取分 离控制系统基本实现了流量的自动控制与集中调整, 即操作人员在控制室中设置流量，现场流量控制设备 即可相对准确地执行控制指令. 同时，在线检测设备 如压力、比重、 $\mathrm{pH}$ 等传感器以及在线稀土纯度分析 等新的技术手段也逐渐进入应用领域. 目前的稀土分 离控制系统中，在线检测手段可比作人的“眼晴”，流 量执行设备可比作人的“手脚”，整个控制系统能够摆 脱对人工设定参数的依赖, 还需要“大脑”, 也就是指 挥稀土萃取分离控制过程的专家系统.

稀土萃取分离过程是典型的非线性、大滞后系 统, 对于分离过程的控制, 有限监测点的在线分析数据 难于简单预测分离过程的运行状态和变化趋势, PID 等通用控制算法也难以得到稳定的控制结果，因此, 稀土分离自动控制系统需要根据稀土萃取分离过程的 特性开发具有针对性的槽体运行状态诊断和调整控制 方法. 目前开发的专家系统主要包括以下内容:

(1)针对特定的稀土分离工艺段, 通过串级萃取理 论静态计算方法得到其最佳平衡态，即在运行试剂消 耗与产品品质稳定性之间优化后的最佳状态，作为控 制的目标.

(2)根据萃取平衡与物料平衡关系，以相关控制点 的分析数据结合流量数据，模拟得到萃取线当前实际 运行状态，再将运行状态与最佳平衡态比较得到的偏
差数据作为方案调整的量化依据.

(3)进一步应用动态仿真技术，从当前运行状态开 始, 可以对不同的调整方案进行推演, 对调整效果及相 应的大滞后、非线性效应进行预判, 优选出最佳调整 方案.

\section{5 分离工序的联动一体化}

对于一个给定的多组分稀土料液, 联动萃取技术 及相关理论的发展已能够计算得到实现其全分离所需 最小消耗的理论极限, 并可设计得到接近理论极限消 耗的工业分离流程，因而单就萃取分离过程而言通过 流程优化设计降低酸碱消耗空间已很有限.

除萃取分离工序外，稀土分离过程还包含原料的 前处理. 我国包头混合型轻稀土矿前处理主体流程为 硫酸法，硫酸浸出液还需萃取转型为氯化物体系后进 行后续的萃取分离. 南方离子吸附型混合碳酸稀土或 氧化稀土矿原料需以盐酸溶解得到后续萃取分离所需 的氯化稀土溶液. 因此, 使用酸性萃取剂的稀土分离过 程存在与包头矿和南方离子吸附型稀土矿原料溶解联 动进行, 从而降低总体酸碱消耗的可能. 基于此联动一 体化思想, 已开展以下工作：

(1)包头矿转型-分组一体化和转型-分离一体化. 包头矿萃取转型及后续的萃取分离分别产生消耗，主 体工艺总萃取量(即所用萃取剂总流量与料液流量之 比)为 3.0 左右. 而如采用转型-分组一体化流程, 在转型 过程中利用萃取剂P204的分离能力同时实现分组，再 进一步在P507联动萃取分离生产线上实现全分离，则 流程的总萃取量可降至1.7左右，在酸碱消耗等方面与 转型后氯化稀土依次全分离工艺相比优势明显.

进一步对包头稀土矿硫酸稀土转型及分离过程的 理论研究获得了避免钙元素在转型过程中富集的工艺 条件，进而设计形成了包头矿转型-分离一体化流程， 即在硫酸稀土萃取转型过程中得到部分纯产品, 实现 分离目的，如图4所示，新流程的总萃取量降至约 1.35 1.40. 上述两工艺在国内某大型稀土分离企业已 成功获得了工业应用, 较大幅度降低了化工试剂消耗.

(2)离子吸附型稀土矿溶料-萃取分离一体化. 基于 非恒定混合萃取比体系串级萃取理论研究，结合不同 稀土元素氧化物碱性差异，设计了在南方离子吸附型 中钎富铕矿溶料所需盐酸消耗量的前提下完成该矿种 


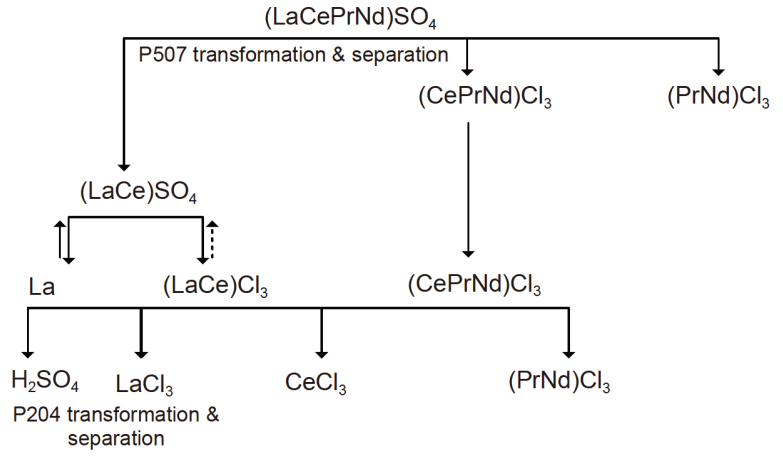

图 4 包头矿稀土转型-分离一体化流程

Figure 4 A process coupled with transformation and separation for Baotou RE mine.

中的钎、铒、铥、镱、镥等重稀土分离的酸性P507 体系萃取分离流程. 一体化工艺效果主要体现在两个 方面，一是新流程中做到了“一酸两用”，即原本溶矿 的酸同时用于有机相再生和溶矿，二是原料中的混合 稀土氧化物间接提供了萃取分离过程所需消耗的碱.
该技术目前正在开展工业化实施.

\section{6 总结与展望}

经过几代稀土工作者跨越半个多世纪的努力，我 国已经从稀土资源大国逐渐发展为稀土生产和应用大 国. 进入二十一世纪以来, 我国稀土分离技术又呈现了 新的发展趋势和特点. 联动萃取理论和技术的应用不 仅大幅降低了稀土分离过程的酸碱消耗和盐排放水 平, 也使稀土溶剂萃取分离流程呈现连续色层化. 杂质 污染的成因分析和控制技术的发展突破了溶剂萃取法 原有纯化瓶颈，已可生产得到更高纯度(如：99.999 99.9999\%)的稀土氧化物产品. 稀土萃取分离生产过程 的智能化是大势所趋，当前的核心任务是利用稀土分 离仿真和自动控制软件方面的优势开发自动控制所需 专家系统. 近年, 串级萃取理论和稀土分离技术虽已取 得一定进展，但对于稀土分离流程的高效、绿色和高 值化仍将是我们继续追求的目标.

\section{参考文献}

1 倪起峰. 稀有金属与硬质合金, 1990, 1: 32-34

2 王学正. 江西有色金属, 1992, 2: 65-67

3 Xu GX. in Rare Earth. 2nd Edition. Beijing: Metallurgical Industry Press, 2005 (in Chinese) [徐光宪. 稀土(上). 第二版. 北京: 冶金工业出版社, 2005]

4 Hsu KH. Acta Scientiarum Naturalium Universitatis Pekinensis, 1978, 1: 51-66 (in Chinese) [徐光宪. 北京大学学报(自然科学版), 1978, 1: 5166]

5 Hsu KH. Acta Scientiarum Naturalium Universitatis Pekinensis, 1978, 1: 67-75 (in Chinese) [徐光宪. 北京大学学报(自然科学版), 1978, 1: 6775]

6 Li PK, Hsu HY, Hsu KH. Acta Scientiarum Naturalium Universitatis Pekinensis, 1980, 2: 66-84 (in Chinese) [李标国, 徐献瑜, 徐光宪. 北京大 学学报(自然科学版), 1980, 2: 66-84]

7 Li BG, Yan CH, Qi SP, Xu GX. J Chin Rare Earth Soc, 1985, 3: 20-26 (in Chinese) [李标国, 严纯华, 乔书平, 徐光宪. 中国稀土学报, 1985, 3: 20-26]

8 Li BG, Yan CH, Qiao SP, Xu GX. J Rare Earths, 1986, 4: 1-7 (in Chinese) [李标国, 严纯华, 乔书平, 徐光宪. 中国稀土学报, 1986, 4: 1-7]

9 Yan CH. One-step scale-up of rare earth extraction separation process. Doctoral Dissertation. Beijing: Graduate School of Peking University, 1988 (in Chinese) [严纯华. 稀土萃取分离工艺的一步放大. 博士学位论文. 北京: 北京大学研究生院, 1988

10 Xu GX, Li BG, Yan CH. Chin Rare Earths, 1985, 1: 56-67 (in Chinese) [徐光宪, 李标国, 严纯华. 稀土, 1985, 1: 56-67]

11 廖春生, 严纯华, 高松, 李标国, 徐光宪. 非恒定混合萃取比稀土分离体系的研究I. 第三届学术年会会议论文I分册, 北京: 中国稀土学会, 1994, 297-301

12 Liao CS. Extraction separation of heavy rare earths, Doctoral Dissertation. Beijing: Graduate School of Peking University, 1996 (in Chinese) [廖 春生. 重稀土的萃取分离, 博士学位论文. 北京:北京大学研究生院, 1996]

13 廖春生, 李标国, 贾江涛, 徐光宪. P507体系与环烷酸分离体系的工艺衔接一中钇富铕矿富钇重稀土料液制备. 见: 稀土化学及湿法治金专 业委员会第五次学术会议论文集, 北京: 中国稀土学会, 1992, VII-1-VII-4 
14 冯艳伟, 何新秀, 汪源成. 成都理工学院学报增刊, 1996, 23: 124-132

15 Wu S, Liao CS, Jia JT, Yan CH. J Chin Rare Earth Soc, 2004, 22: 17-21 (in Chinese) [吴声, 廖春生, 贾江涛, 严纯华. 中国稀土学报, 2004, 22: $17-21]$

16 Wu S, Liao CS, Jia JT. Yan CH. J Chin Rare Earth Soc, 2004, 22: 171-176 (in Chinese) [吴声, 廖春生, 贾江涛, 严纯华. 中国稀土学报, 2004, 22: 171-176]

17 Wu S, Liao CS, Yan CH. J Chin Rare Earths, 2012, 30: 163-167 (in Chinese) [吴声, 廖春生, 严纯华. 中国稀土学报, 2012, 30: 163-167]

18 Yan CH, Wu S, Liao CS, Jia JT, Cheng FX, Wang SL, Zhang B. Chin J Inorg Chem, 2008, 24: 1200-1205 (in Chinese) [严纯华, 吴声, 廖春生, 贾江涛, 程福祥, 王嵩龄, 张玻. 无机化学学报, 2008, 24: 1200-1205]

19 Liao CS, Cheng FX, Wu S, Yan CH. J Chin Rare Earths, 2017, 35: 1-8 (in Chinese) [廖春生, 程福祥, 吴声, 严纯华. 中国稀土学报, 2017, 35: $1-8]$

20 Liao C, Wu S, Cheng F, Wang S, Liu Y, Zhang B, Yan C. J Rare Earths, 2013, 31: 331-336

21 Cheng FX, Wu S, Liao CS, Yan CH. J Chin Rare Earths, 2018, 36: 292-300 (in Chinese) [程福祥, 吴声, 廖春生, 严纯华. 中国稀土学报, 2018, 36: 292-300]

22 Cheng FX, Wu S, Liao CS, Yan CH. J Chin Rare Earths, 2018, 36: 437-449 (in Chinese) [程福祥, 吴声, 廖春生, 严纯华. 中国稀土学报, 2018, 36: 437-449]

23 Cheng FX, Wu S, Liao CS, Yan CH. J Chin Rare Earths, 2018, 36: 571-582 (in Chinese) [程福祥, 吴声, 廖春生, 严纯华. 中国稀土学报, 2018, 36: 571-582]

24 Cheng FX, Wu S, Liao CS, Yan CH. J Chin Rare Earths, 2018, 36: 672-681 (in Chinese) [程福祥, 吴声, 廖春生, 严纯华. 中国稀土学报, 2018, 36: 672-681]

25 Cheng FX, Wu S, Liao CS, Yan CH. J Chin Rare Earths, 2019, 37: 39-48 (in Chinese) [程福祥, 吴声, 廖春生, 严纯华. 中国稀土学报, 2019, 37: 39-48]

26 Cheng FX, Wu S, Liao CS, Yan CH. J Chin Rare Earths, 2019, 37: 199-209 (in Chinese) [程福祥, 吴声, 廖春生, 严纯华. 中国稀土学报, 2019, 37:199-209]

27 Wu S, Liao CS, Yan CH. J Chin Rare Earths, 2012, 30: 163-167 (in Chinese) [吴声, 廖春生, 严纯华. 中国稀土学报, 2012, 30: 163-167] 


\title{
Trends and progress of the theory of countercurrent extraction and rare earth separation technology
}

\author{
Chun-Sheng Liao ${ }^{1,2^{*}}$, Fu-Xiang Cheng ${ }^{1,2}$, Sheng $\mathrm{Wu}^{1,2}$, Chun-Hua Yan ${ }^{2}$ \\ ${ }^{1}$ China Minmetals (Beijing) Research Institute of RE Co., Ltd, Beijing 100085, China; \\ ${ }^{2}$ College of Chemistry and Molecular Engineering of Peking University, State Key Laboratory of Rare Earth Materials Chemistry and Applications, \\ Beijing National Laboratory for Molecular Sciences, Beijing 100871, China \\ *Corresponding author (email: liao@cre-ol.com)
}

\begin{abstract}
Rare earths are significant for new material developments. China has the largest proved reserve of rare earths, and developed leading theories and technologies of rare earth extraction separation in the world. To adapt to the new requirements of environment protect and intelligent manufacturing in the last two decades, rare earth separation technologies behave the development trends of continuous chromatography, higher product purity, automatic control and process coupling. A flow-sheet with hyperlink extraction technology presents the separation efficiency as continuous ion exchange chromatography. Improvements on cause-effect analysis and control technologies of cross contamination during the process deepened the purification limitation by extraction separation. Automatically controlled production based on a special simulation expert system for flow-sheet optimization design will provide effective guarantee for the quality and stability of rare earth products. The coupling of extraction separation with other processes has proved to be a new idea to further improve green manufacturing level of rare earth separation. Herein, in combination with our recent studies, we have introduced the trends and progress of the theory of countercurrent extraction and rare earth separation technology.
\end{abstract}

Keywords: theory of countercurrent extraction, rare earth separation, high-purity rare earths, automatic control, process coupling

doi: $10.1360 /$ SSC-2020-0134 